\title{
BMJ Open Risk factors for malignant transformation of hepatocellular adenoma to hepatocellular carcinoma: protocol for systematic review and meta-analysis
}

\author{
Tharusan Thevathasan, ${ }^{1}$ Teresa Colbatzky, ${ }^{2}$ Moritz Schmelzle, ${ }^{2}$ Johann Pratschke, ${ }^{2}$ \\ Felix Krenzien (1) ${ }^{2}$
}

To cite: Thevathasan $\mathrm{T}$, Colbatzky T, Schmelzle M, et al. Risk factors for malignant transformation of hepatocellular adenoma to hepatocellular carcinoma: protocol for systematic review and meta-analysis. BMJ Open 2021;11:e045733. doi:10.1136/ bmjopen-2020-045733

- Prepublication history and additional supplemental material for this paper are available online. To view these files, please visit the journal online. (http://dx.doi.org/10.1136/ bmjopen-2020-045733).

Received 09 0ctober 2020 Accepted 24 July 2021

Check for updates

(c) Author(s) (or their employer(s)) 2021. Re-use permitted under CC BY-NC. No commercial re-use. See rights and permissions. Published by BMJ.

${ }^{1}$ Department of Cardiology, Charité - Universitätsmedizin Berlin, Berlin, Germany

${ }^{2}$ Department of Surgery, Charité - Universitätsmedizin Berlin, Berlin, Germany

Correspondence to

Dr Felix Krenzien;

felix.krenzien@charite.de

\section{ABSTRACT}

Introduction Hepatocellular adenomas (HCAs) are solid liver tumours that are usually found incidentally during routine medical check-ups. Multiple modifiable and non-modifiable factors constitute a risk for the malignant transformation of HCAs to hepatocellular carcinoma ( $\mathrm{HCC}$ ), which has emerged to be one of the fastest growing causes of cancer-related mortality globally. This study protocol for a planned systematic review and meta-analysis documents the methodological approach to identify risk factors and their risk estimates for the transformation from HCA to HCC.

Methods and analysis Two independent reviewers will systematically search and extract data from studies in patients of all ages published between January 1970 and June 2021 on PubMed, MEDLINE, EMBASE, Cumulative Index to Nursing and Allied Health Literature, Scopus Web of Science, Ovid, The Cochrane Hepatobiliary Group Controlled Trials Register and The Cochrane Central Register of Controlled Trials by using an a priori defined search strategy. Study quality will be rated with the National Institute of Health quality assessment tools. Disagreements will be resolved by consensus with a third independent reviewer. The primary outcome will be the odds ratio (OR) of developing HCC in patients with prediagnosed HCA depending on the exposure to risk factors. HCC diagnosis must be inferred based on imaging techniques or pathology. We will use R V.4.0.2 to conduct meta-analyses and generate pooled ORs based on random effects models. Results will be presented as forest plots. Cochran's $Q$ and $I^{2}$ test will be performed to assess heterogeneity between included studies. Funnel plots and Egger's weighted regression will be used to evaluate publication bias.

Ethics and dissemination № ethical approval is required as we will use and analyse data from previously published studies in which informed consent was obtained. The results will be disseminated in a peer-reviewed journal on completion. PROSPERO registration number CRD42020206578.

\section{INTRODUCTION}

\section{Epidemiology of hepatocellular adenoma}

Hepatocellular adenomas (HCAs) are benign, solid liver tumours that are usually asymptomatic and found incidentally during routine medical check-ups. Being presumably first characterised by Frerichs in $1861,{ }^{1}$ HCAs have gained wide recognition since the 1960s
Strengths and limitations of this study

- Despite the global rise in liver cancer-related mortality and the potential of hepatocellular adenoma (HCA) for malignant transformation, management strategies for HCA are vaguely defined with regards to conservative versus surgical management based on patient risk factors.

- To the best of our knowledge, this is the first metaanalysis on identifying risk factors as well as their risk estimates for malignant transformation from HCA to hepatocellular carcinoma.

- This extensive systematic review and meta-analysis will include publications from 1970 to 2021, which will be reviewed and rated by three independent reviewers according to established quality assessment instruments.

- Malignant transformation from HCA to hepatocellular carcinoma may not be subjected to a single risk factor (eg, tumour size) -multifactorial carcinogenesis, including genetic mutations, is more likely.

and 1970s with the introduction of oral contraceptive pills (OCPs), one of the most established risk factors for HCA tumourigenesis in women of childbearing age ${ }^{2-4}$ The annual incidence of HCA in women with prolonged OCP intake is approximately 3-4 per 100000 OCP users. ${ }^{5}$ The understanding of HCA risk factors has evolved gradually, which include demographic factors (eg, male sex), environmental factors (eg, anabolic steroid intake, obesity or metabolic syndrome), underlying liver diseases or genetic syndromes, such as glycogen storage diseases or familial adenomatous polyposis.

\section{Malignant transformation to hepatocellular carcinoma}

Given their potential for malignant transformation (hepatocellular carcinogenesis), HCAs constitute a risk for developing hepatocellular carcinoma (HCC) or haemorrhage, 
which may be accompanied by abdominal pain, distension or displacement of surrounding structures. Malignant transformation to HCC has been reported in $4.2 \%$ of patients with HCA. ${ }^{5}$

Multiple risk factors for the transformation to HCC have been identified, ${ }^{15}$ such as male sex, progression of HCA size, exogenous androgen or oestrogen intake, as well as genetic predispositions, such as mutations in b-catenin gene, hepatocyte nuclear factor 1 alpha gene or glycogen storage diseases.

On a global scale, liver cancer has become one of the fastest growing causes of cancer-related death, ${ }^{6}$ with a $43 \%$ increase in mortality rate in the USA between 2000 and 2016. ${ }^{7}$ As of 2018, HCC was the sixth most commonly diagnosed cancer in both sexes worldwide, as well as the second most common cause of cancer-related mortality. ${ }^{8}$

\section{Rationale for systematic review and meta-analysis}

Despite the aforementioned global trend ${ }^{6-8}$ and HCA's potential for malignant transformation, management strategies for HCA are vaguely defined. Management strategies include liver resection vs conservative therapy (ie, life style change and wait and watch). There appears to be one systematic review from 2010 evaluating the risk factors for malignant transformation from HCA to HCC to the best of our knowledge. ${ }^{5}$ Since then, numerous studies have been published on risk factors for the transformation from HCA to HCC, in particular including HCA molecular subtypes and genetic mutations. ${ }^{9-13}$ Further, there appears to be an evidence gap for the quantitative risk assessment for the malignant transformation from HCA to HCC based on patients' underlying risk factors.

In fact, sex and size of HCA remain the most wellestablished parameters for treatment decisions in accordance with the guidelines of The European Association for the Study of the Liver. Elective liver resection is recommended in all men, while women are subjected to surgery when HCA diameter is greater than $5 \mathrm{~cm}$ and/or size has been progressing despite life-style changes. ${ }^{14} 15$ Nonetheless, multiple cases reports showed malignant transformation in HCAs with diameters $<5 \mathrm{~cm}$, while other studies suggest an elective surgery for diameters $>4$ or $>8 \mathrm{~cm}$, opening a debate for clear cut-off values for surgical treatment. ${ }^{16-18}$ Selected patients obviously benefit from a conservative therapy, such as the cessation of exogenous hormone intake, while others may benefit from surgery. ${ }^{19} 20$

Therefore, the aim of this study protocol is a systematic review to provide the latest overview on modifiable (eg, exogenous intake of oestrogens or androgens) and non-modifiable risk factors (eg, sex, genetic mutations or molecular subtypes of HCA) for the malignant transformation from HCA to HCC in patients of all ages. If different molecular subtypes are present in the same patient, we will include the patient in subgroups for each given molecular subtype. In addition, we aim to conduct a meta-analysis to provide risk estimates for the transformation from HCA to HCC in patients who have been exposed to a risk factor-in comparison to patients without exposure to the same risk factor.

By assessing which patient with HCA harbours the highest risk for HCC genesis, our data may support risk factor-adjusted patient management and therapy (surgery vs surveillance) - with the overall aim to improve patient outcomes and prevent malignant transformation.

\section{METHODS AND ANALYSIS}

We will conduct a systematic review and meta-analysis between June 2021 and October 2021. The development of the study design, study conduct and reporting of results will be in accordance to the Preferred Reporting Items for Systematic Reviews and Meta Analyses Protocols (PRISMA) (see online supplemental material 1) and Meta-analysis of Observational Studies in Epidemiology (MOOSE) guidelines. ${ }^{21} 22$

\section{Objectives}

To conduct a systematic review and meta-analysis to investigate modifiable and non-modifiable risk factors of HCA, as well as their risk estimate for the malignant transformation to HCC for patients of all ages in the published literature.

\section{Study questions}

- The systematic review will address the following question: What are modifiable and non-modifiable risk factors for the malignant transformation of HCA to HCC in the literature published from 1970 to 2021?

- The meta-analysis will address the following question: What is the quantitative risk estimate for the malignant transformation of HCA to HCC in patients who have been exposed to a specific risk factor-compared with patients without exposure to the same risk factor?

\section{Eligibility criteria}

Inclusion and exclusion criteria are defined as follows (see figure 1 for PRISMA flow diagram):

\section{Inclusion criteria}

- Randomised controlled trials, observational studies (ie, cross-sectional, case-control or cohort studies with a prospective or retrospective study design) and case reports, which reported the frequency of malignant transformation from HCA to HCC in adults and non-adults.

- HCA and HCC were diagnosed based on imaging techniques or pathology according to recommendations by the European Association for the Study of the Liver. ${ }^{1415}$

- Studies were published in English or German language from 1 January 1970 to 1 June 2021, regardless of the country or ethnic background of the study population. We selected this time period as the first known reports were published in the 1970s. 


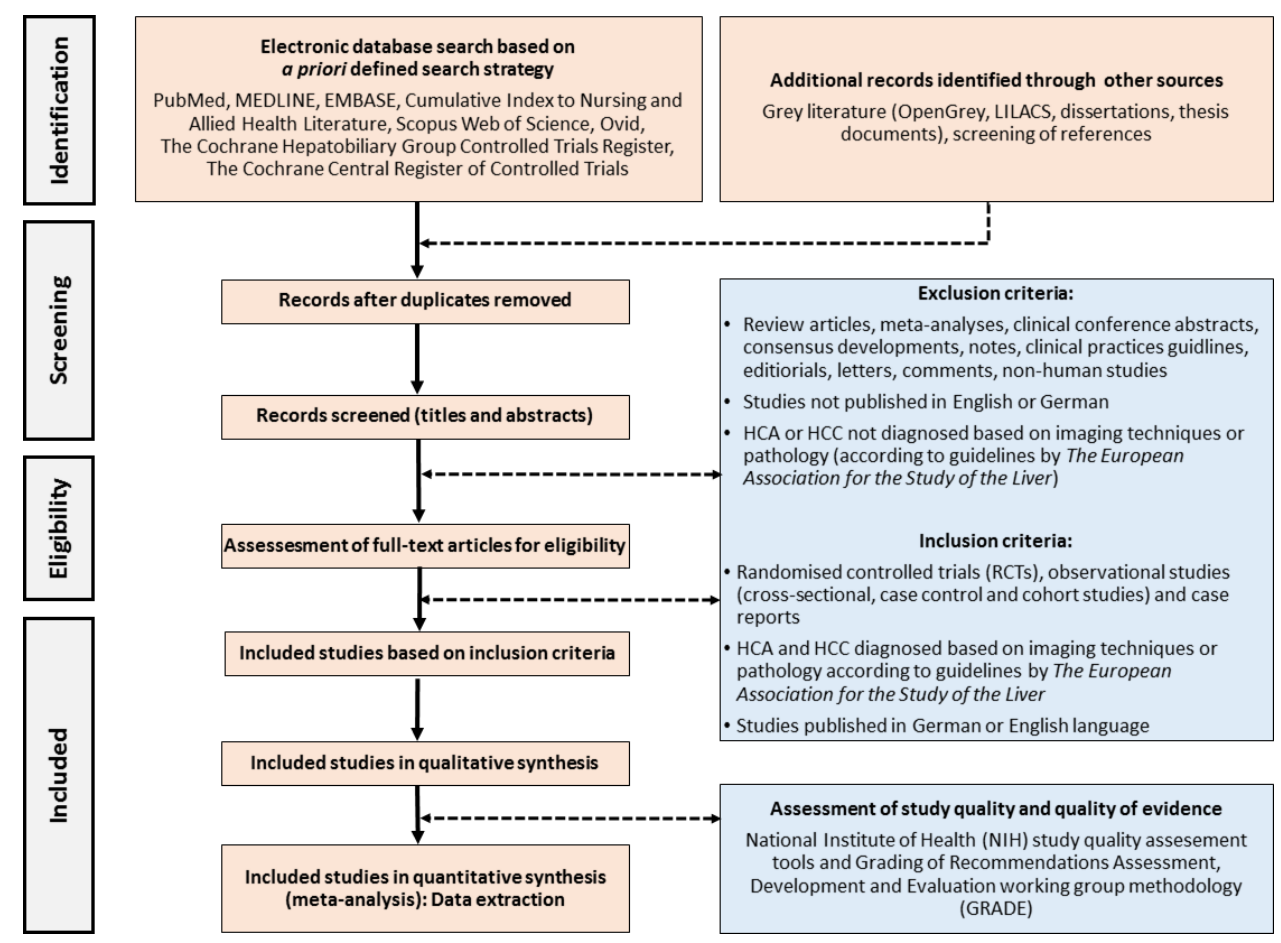

Figure 1 PRISMA flow diagram for systematic review and meta-analysis on malignant transformation from hepatocellular adenoma (HCA) to hepatocellular carcinoma (HCC). PRISMA, Preferred Reporting Items for Systematic Reviews and MetaAnalyses.

- In case of multiple publications of the same data, we will consider the most comprehensive, recent and inclusive study.

\section{Exclusion criteria}

- (Systematic) review articles and meta-analyses to allow for an independent literature review without a study selection bias.

- Clinical conference abstracts, consensus development, notes, clinical practice guidelines, editorials, letters and comments.

- Studies with unclear reporting of risk estimates for malignant transformation from HCA to HCC.

- Studies in which HCA and HCC were not diagnosed based on imaging techniques or pathology according to recommendations by the European Association for the Study of the Liver. ${ }^{14} 15$

- Non-human studies.

- Studies were not published in English or German.

\section{Search strategy}

We will conduct a comprehensive literature search to identify relevant studies according to our aforementioned eligibility criteria. We will perform a systematic search on PubMed, MEDLINE, EMBASE, Cumulative Index to Nursing and Allied Health Literature (CINAHL), Scopus Web of Science, Ovid, The Cochrane Hepatobiliary Group Controlled Trials Register and The Cochrane Central Register of Controlled Trials (CENTRAL) by using an a priori defined search strategy based on a combination of keywords of our research question: 'hepatocellular adenoma' and 'patients'. In contrast to a previously published systematic review, ${ }^{5}$ we will not include the keywords 'malignant transformation' or 'liver resection' to allow for a more open search approach. For grey literature, we will use OpenGrey, LILACS, dissertations or thesis documents.

We will conduct the search based on principles of Boolean operators (AND, OR and NOT), incorporate free-text words and Medical Subject Headings, as well as different versions of medical terminology. We will use the following search strategy on PubMed:

("hepatic" OR "liver" OR "liver cell" or "hepatocellular") AND ("adenoma" or "hepatoma") AND ("patient") NOT (meta-analysis[Filter]) NOT (review[Filter]) NOT (systematicreview[Filter]) NOT (comment[Filter]) NOT (congress[Filter]) NOT (consensusdevelopmentconference[Filter]) NOT (consensusdevelopmentconferencenih[Filter]) NOT (editorial[Filter]) NOT (guideline[Filter]) NOT (letter[Filter]) AND (humans[Filter]) AND (english[Filter] OR german[Filter]) AND (1970:2021[pdat])

\section{Study selection}

Reviewer A will apply the search strategy across all aforementioned electronic platforms and compile a list of retrieved titles in Microsoft Excel. Independent investigators $\mathrm{B}$ and $\mathrm{C}$ will review the listed publications by screening their titles and abstracts for eligibility criteria. After screening, the full text of all eligible studies deemed relevant will be retrieved and again independently reviewed deliberately by reviewers B and C to ascertain eligibility. In case of disagreement, consensus will be obtained by 


\begin{tabular}{|c|c|c|c|}
\hline \multirow{2}{*}{ Odds ratic } & & \multicolumn{2}{|c|}{$\begin{array}{c}\text { HCC in patients with } \\
\text { pre-diagnosed HCA }\end{array}$} \\
\hline & & $\begin{array}{c}\text { Yes } \\
\text { (Case) }\end{array}$ & $\begin{array}{c}\text { No } \\
\text { (Control) }\end{array}$ \\
\hline \multirow{2}{*}{$\begin{array}{l}\text { Exposure to } \\
\text { risk factor }\end{array}$} & Yes & $\mathrm{a}$ & $\mathrm{b}$ \\
\hline & No & $\mathrm{c}$ & $\mathrm{d}$ \\
\hline
\end{tabular}

Figure 2 Calculation of ORs of developing hepatocellular carcinoma (HCC) in patients with prediagnosed hepatocellular adenoma ( $\mathrm{HCA})$ based on exposure to risk factors.

reviewer $\mathrm{A}$. In addition, $\mathrm{B}$ and $\mathrm{C}$ will complement the database search by manually reviewing the reference lists of all included publications to include publications that may have not been identified by the search strategy.

\section{Data extraction and management}

Two data extractors will independently extract the data from all included studies by using a standardised data collection form which will be created by a third reviewer. The following data will be extracted: authors, year, country, study design, setting, sample size, risk factor, frequency of HCC in HCA patients with exposure to a risk factor, frequency of HCC in HCA patients without exposure to a risk factor, frequency without HCC in HCA patients with exposure to a risk factor, frequency without HCC in HCA patients without exposure to a risk factor (see paragraph on the section 'Primary Outcome'). Interrater agreement between the two data extractors will be analysed using kappa statistics. ${ }^{23}$

If the frequency of HCA or HCC is not reported, we will calculate the frequency using the study sample size and the number of outcomes. In studies where HCC incidence is reported in two or more arms, each study arm will be considered as a single study. In this case, we will extract the data from each study arm separately.

\section{Missing outcome data}

In case of missing outcome data, we will contact the corresponding study authors. We will perform sensitivity analyses to assess the robustness of the meta-analysis and discuss the impact of missing data on the findings. We will not apply statistical approaches (eg, data imputation, analysis of worst and best cases) to adjust for missing

Figure 3 Planned meta-analyses (based on sample data as an example) on risks of developing hepatocellular carcinoma (HCC) in patients with prediagnosed hepatocellular adenoma (HCA) depending on exposure to risk factors. 
outcome data as these are considered to be not effective and less recommended in meta-analyses. ${ }^{24}$

\section{Methodological study quality and quality of evidence}

The methodological quality (risk of bias) of the included interventional and observational studies will be rated by two independent reviewers as good, fair and poor by incorporating the National Institute of Health quality assessment tools. ${ }^{25}$ Additionally, the strength of the body of evidence will be rated as very low, low, moderate and high by using the Grading of Recommendations Assessment, Development and Evaluation working group methodology. ${ }^{26}$ We will assess the quality of evidence across the domains of risk of bias, consistency, directness, precision and publication bias.

\section{Primary outcome}

The primary outcome of our meta-analysis will be the OR of developing HCC in HCA patients based on as to whether the patients were exposed to a risk factor. Diagnosis of HCC must be inferred based on imaging techniques or pathology. ${ }^{15}$ We will collect the following information to calculate the odds ratio (see figure 2):

1. Number of HCA patients who were exposed to a risk factor and have developed HCC.

2. Number of HCA patients who were exposed to a risk factor and have not developed HCC.

3. Number of HCA patients who were not exposed to a risk factor and have developed HCC.

4. Number of HCA patients who were not exposed to a risk factor and have not developed HCC.

\section{Data synthesis, results presentation and statistical analyses}

Number of included and excluded search results will be presented in a PRISMA flow diagram, including the rationale for study exclusion (see figure 1$) \cdot{ }^{27}$ Included studies will be presented in an evidence table and summarised by using descriptive statistics. For studies that are not eligible for quantitative synthesis, we will provide a narrative synthesis with information being presented in the text and/or a table to summarise and explain the characteristics and findings.

We will use R V.4.0.2 (R Core Team 2020. Vienna, Austria) to conduct the meta-analyses and generate a pooled risk estimate of the overall OR for developing in HCC in patients with prediagnosed HCA depending on exposure to risk factors (see figure 3 ).

\section{Study heterogeneity}

We will assess the study heterogeneity between publications by using the Cochran's $Q$ test and $I^{2}$ statistics. We will define heterogeneity as low, moderate and high in accordance to values below $25 \%$, between $25 \%$ and $75 \%$ and above $75 \%$, respectively. We will apply random effects models to account for heterogeneity in meta-analyses, as well as random effects meta-regression analysis to examine the sources of heterogeneity.
Subgroup analyses and cumulative meta-analysis

We will conduct subgroup analyses based on different HCA diameters, study quality (high-quality vs low-quality studies) and time period (prior to 2000 vs post-2000). With an exploratory intent, we will perform a cumulative meta-analysis to identify the time point at which the risk factors first reached conventional levels of significance.

\section{Publication bias}

We will use funnel plots and Egger's weighted regression to assess publication bias. In case of existing publication bias, we will perform Duval and Tweedie non-parametric 'trim-and-fill' analyses.

\section{Twitter Tharusan Thevathasan @TharusanT}

Contributors TT, TC, MS, JP and FK have made substantial contributions to the conception and design of the planned systematic review and meta-analysis, as well as have written, critically revised and approved the study protocol. FK is the guarantor of the systematic review and meta-analysis. All authors meet the ICMJE criteria for authorship.

Funding The authors have not declared a specific grant for this research from any funding agency in the public, commercial or not-for-profit sectors.

Competing interests None declared.

Patient consent for publication Not required.

Provenance and peer review Not commissioned; externally peer reviewed.

Supplemental material This content has been supplied by the author(s). It has not been vetted by BMJ Publishing Group Limited (BMJ) and may not have been peer-reviewed. Any opinions or recommendations discussed are solely those of the author(s) and are not endorsed by BMJ. BMJ disclaims all liability and responsibility arising from any reliance placed on the content. Where the content includes any translated material, BMJ does not warrant the accuracy and reliability of the translations (including but not limited to local regulations, clinical guidelines, terminology, drug names and drug dosages), and is not responsible for any error and/or omissions arising from translation and adaptation or otherwise.

Open access This is an open access article distributed in accordance with the Creative Commons Attribution Non Commercial (CC BY-NC 4.0) license, which permits others to distribute, remix, adapt, build upon this work non-commercially, and license their derivative works on different terms, provided the original work is properly cited, appropriate credit is given, any changes made indicated, and the use is non-commercial. See: http://creativecommons.org/licenses/by-nc/4.0/.

ORCID iD

Felix Krenzien http://orcid.org/0000-0003-0593-9917

\section{REFERENCES}

1 Deneve JL, Pawlik TM, Cunningham S, et al. Liver cell adenoma: a multicenter analysis of risk factors for rupture and malignancy. Ann Surg Oncol 2009;16:640-8.

2 Baum JK, Bookstein JJ, Holtz F, et al. Possible association between benign hepatomas and oral contraceptives. Lancet 1973;2:926-9.

3 Edmondson HA, Henderson B, Benton B. Liver-Cell adenomas associated with use of oral contraceptives. N Engl J Med Overseas Ed 1976;294:470-2.

4 Rooks JB, Ory HW, Ishak KG, et al. Epidemiology of hepatocellular adenoma. The role of oral contraceptive use. JAMA 1979;242:644-8.

5 Stoot JHMB, Coelen RJS, De Jong MC, et al. Malignant transformation of hepatocellular adenomas into hepatocellular carcinomas: a systematic review including more than 1600 adenoma cases. HPB 2010;12:509-22.

6 Villanueva A. Hepatocellular carcinoma. N Engl J Med 2019;380:1450-62.

$7 \mathrm{Xu} \mathrm{J}$. Trends in liver cancer mortality among adults aged 25 and over in the United States, 2000-2016. NCHS Data Brief 2018:1-8.

8 Bray F, Ferlay J, Soerjomataram I, et al. Global cancer statistics 2018: GLOBOCAN estimates of incidence and mortality worldwide for 36 cancers in 185 countries. CA Cancer J Clin 2018;68:394-424. 
9 Zheng J, Sadot E, Vigidal JA, et al. Characterization of hepatocellular adenoma and carcinoma using microRNA profiling and targeted gene sequencing. PLoS One 2018;13:e0200776.

10 Nault J-C, Couchy G, Balabaud C, et al. Molecular Classification of Hepatocellular Adenoma Associates With Risk Factors, Bleeding, and Malignant Transformation. Gastroenterology 2017;152:880-94.

11 Krause K, Tanabe KK. A shifting paradigm in diagnosis and management of hepatic adenoma. Ann Surg Oncol 2020;27:3330-8.

12 Sciarra A, Schmidt S, Pellegrinelli A, et al. OATPB1/B3 and MRP3 expression in hepatocellular adenoma predicts Gd-EOBDTPA uptake and correlates with risk of malignancy. Liver Int 2019;39:158-67.

13 Longerich T, Endris V, Neumann O, et al. RSPO2 gene rearrangement: a powerful driver of $\beta$-catenin activation in liver tumours. Gut 2019;68:1287-96.

14 European Association for the Study of the Liver (EASL). EASL clinical practice guidelines on the management of benign liver tumours. $J$ Hepatol 2016;65:386-98.

15 European Association for the Study of the Liver. Electronic address: easloffice@easloffice.eu, European Association for the Study of the Liver. EASL clinical practice guidelines: management of hepatocellular carcinoma. J Hepatol 2018;69:182-236.

16 Dokmak S, Paradis V, Vilgrain V, et al. A single-center surgical experience of 122 patients with single and multiple hepatocellular adenomas. Gastroenterology 2009;137:1698-705.

$17 \mathrm{Kim} \mathrm{DH}$, Kim SU, Nam DH, et al. A case of hepatocellular carcinoma within hepatocellular adenoma in a non-cirrhotic male. Korean $\mathrm{J}$ Intern Med 2009;24:147-52.
18 Micchelli STL, Vivekanandan P, Boitnott JK, et al. Malignant transformation of hepatic adenomas. Mod Pathol 2008;21:491-7.

19 Haring MPD, Gouw ASH, de Haas RJ, et al. The effect of oral contraceptive pill cessation on hepatocellular adenoma diameter: a retrospective cohort study. Liver Int 2019;39:905-13.

20 Klompenhouwer AJ, Bröker MEE, Thomeer MGJ, et al. Retrospective study on timing of resection of hepatocellular adenoma. $\mathrm{Br} J$ Surg 2017;104:1695-703.

21 Moher D, Shamseer L, Clarke M, et al. Preferred reporting items for systematic review and meta-analysis protocols (PRISMA-P) 2015 statement. Syst Rev 2015;4:1.

22 Stroup DF, Berlin JA, Morton SC, et al. Meta-Analysis of observational studies in epidemiology: a proposal for reporting. meta-analysis of observational studies in epidemiology (moose) group. JAMA 2000;283:2008-12.

23 Viera AJ, Garrett JM. Understanding interobserver agreement: the kappa statistic. Fam Med 2005;37:360-3.

24 Tacconelli E. Systematic reviews: CRD's guidance for undertaking reviews in health care. Lancet Infect Dis 2010;10:226.

25 Study Quality Assessment Tools. Available: https://www.nhlbi.nih. gov/health-topics/study-quality-assessment-tools [Accessed $19 \mathrm{Sep}$ 2020].

26 Balshem H, Helfand M, Schünemann HJ, et al. Grade guidelines: 3. rating the quality of evidence. J Clin Epidemiol 2011;64:401-6.

27 PRISMA. Available: http://www.prisma-statement.org/ PRISMAStatement/FlowDiagram [Accessed 29 Sep 2020]. 